\title{
Medical Image Processing in Nuclear Medicine and Bone Arthroplasty
}

\author{
Joyjit Patra $^{1}$, Himadri Nath Moulick ${ }^{2}$, Arun Kanti Manna ${ }^{3}$ \\ ${ }^{1,2}$ (C.S.E..Aryabhatta Institute Of Engineering And Management,Durgapur,West Bengal,India) \\ ${ }_{3}^{3}$ (C.S.E,Modern Institute of Engineering And Technology,Hoogly, West Bengal,India)
}

\begin{abstract}
Computers have become indispensable in all domains, and the medical segment does not represent an exception. The need for accuracy and speed has led to a tight collaboration between machines and human beings. Maybe the future will allow the existence of a world where the human intervention won't be necessary, but for now, the best approach in the medical field is to create semiautomatic applications, in order to help the doctors with the diagnoses, with following the patients' evolution and managing them and with other medical activities. Our application is designed for automatic measurements of orthopedic parameters, and allows the possibility of human intervention in case the parameters have not been detected properly. The segment of the application is Hip Arthroplasty.
\end{abstract}

Keywords: Hip Arthroplasty, Canny Edge Detection, DICOM, Hough Transform, Radiographic Image

Processing.

\section{INTRODUCTION}

Medical image processing is an area of increasing interest. It includes a wide range of methods and techniques, starting with the acquisition of images using specialized devices (for example, CT devices), image enhancement and analysis, to 3D model reconstruction from 2D images. Thus, the research in this field represents a point of interest for both doctors and engineers, in their attempt to improve medical techniques, with computer assistance, in order to obtain more accurate results in treating the patients.Among many research projects in this area of interest some of the most relevant are: - The SCANIP [11] image processing software that provides a broad range of image visualization, processing and segmentation tools for medical purposes. This software has been created by Simpleware in Great Britain. The SCANIP programs ensure the conversion of

3D medical images into quality meshes. These meshes can be used in future processing in analysis programs, in fluid dynamics, CAD and the creation of Rapid Prototyping models. The sources for these programs come from MRIs, CTs or MicroCTs.

The 3D-DOCTOR Project [12] that comes with an advanced 3d modeling software, with strong processing and measurement functions for MRI-s, CT-s, PET-s, and other types of medical images.The possible applications of this software are in the scientific and medical domain, but also in the image processing industrial field. The functioning principle of this software is based on edge detection techniques using 3D image segmentation functions and on the construction of 3D surfaces and volumes, that are afterwards visualized and measured for the purpose of a quantitative and qualitative analysis. - The Hip-OpCT software [13] that allows importing CT images in DICOM format. Once imported, theCT dataset is visualized through several modalities from which the doctors can plan the size and the position of the prosthesis. Advanced techniques of image processing and analysis find widespread use in medicine. In medical applications, image data are used to gather details regarding the process of patient imaging whether it is a disease process or a physiological process.

Information provided by medical images has become a vital part of today's patient care. The images generated in medical applications are complex and vary notably from application to application. Nuclear medicine images show characteristic information about the physiological properties of the structures-organs. In order to have high quality medical images for reliable diagnosis, the processing of image is necessary. The scope of image processing and analysis applied to medical applications is to improve the quality of the acquired image and extract quantitative information from medical image data in an efficient and accurate way.MatLab (Matrix Laboratory) is a high performance interactive software package for scientific and engineering computation developed by MathWorks (Mathworks Inc., 2009).MatLab allows matrix computation, implementation of algorithms, simulation, plotting of functions and data, signal and image processing by the Image Processing Toolbox. It enables quantitative analysis and visualisation of nuclear medical images of several modalities, suchas Single Photon Emission Computed Tomography (SPECT), Positron Emission Tomography (PET) or a hybrid system (SPECT/CT) where a Computed Tomography system (CT) is incorporated to the SPECT system. The Image Processing Toolbox (Mathworks Inc., 2009) is a comprehensive set of reference-standard algorithms and graphical tools for image processing, analysis, visualisation and algorithm development. It offers the possibility to restore noisy or degraded images, enhance images for 
improved intelligibility, extract features, analyse shapes and textures, and register two images. Thus, it includes all the functions that MatLab utilises in order to perform any sophisticated analysis needed after the acquisition of an image. Most toolbox functions are written in open MatLab language offering the opportunity to the user to inspect the algorithms, to modify the source code and create custom functions (Wilson et al., 2003, Perutka, 2010).This chapter emphasises on the utility of MatLab in nuclear medicine images' processing. It includes theoretical background as well as examples. After an introduction to the imaging techniques in nuclear medicine and the quality of nuclear medicine images, this chapter proceeds to a study about image processing in nuclear medicine through MatLab. Image processing techniques presented in this chapter include organ contouring, interpolation, filtering, segmentation, background activity removal, registration and volume quantification. A section about DICOM image data processing using MatLab is also presented as this type of image is widely used in nuclear medicine.

\section{NUCLEAR MEDICINE IMAGING}

Nuclear Medicine is the section of science that utilises the properties of radiopharmaceuticals in order to derive clinical information of the human physiology and biochemistry. According to the examination needed for each patient, a radionuclide is attached to a pharmaceutical (tracer) and the whole complex is then delivered to the patient intravenously or by swallowing or even by inhalation. The radiopharmaceutical follows its physiological pathway and it is concentrated on specific organs and tissues for short periods of time. Then, the patient is positioned under a nuclear medicine equipment which can detect the radiation emitted by the human body resulting in images of the biodistribution of the radiopharmaceutical. In Nuclear Medicine, there are two main methods of patient imaging, the imaging with Planar Imaging, Dynamic Imaging or SPECT and the PET.

During the last decade, hybrid systems have been developed integrating the CT technique with either SPECT or PET resulting in SPECT/CT and PET/CT respectively. This chapter will concentrate on the implementation of MatLab code in gamma camera planar imaging, SPECT and SPECT/CT methods. The gamma camera is composed of a collimator, a scintillator crystal usually made of NaI (or CsI), the photomultiplier tubes, the electronic circuits and a computer equipped with the suitable software to depict the nuclear medicine examinations. In planar imaging, the patient, having being delivered with the suitable radiopharmaceutical, is sited under the gamma camera head. The gamma camera head remains stable at a fixed position over the patient for a certain period of time, acquiring counts (disintegrations). These will constitute the radiopharmaceutical distribution image. The counts measured in a specific planar projection originate from the whole thickness of patient (Wernick \& Aarsvold, 2004). In SPECT, the gamma camera head rotates around the patient remaining at well defined angles and acquiring counts for specific periods of time per angle. What makes SPECT a valuable tool in nuclear medicine is the fact that information in the three dimensions of the patient can be collected in a number of slices with a finite known volume (in voxels). Thus, SPECT technique is used to display the radiopharmaceutical distribution in a single slice removing the contribution from the overlying and underlying tissues. In order to obtain the most accurate quantitative data from SPECT images, two issues that have to be resolved are the attenuation correction and the Compton scattering that the photons are undergone until reach and interact with the slice of interest tissues. As an examining organ has certain dimensions, each slice along the axis of the gamma camera has different distance from the detector. Thus, each photon experiences different attenuation. These two phenomena usually lead to distortion of the measured activity concentration (Wernick \& Aarsvold, 2004). The acquired data are processed in order to correct and compensate the undesired effect of these physical phenomena. The projection data of each slice constitute the sinogram. As a result, a series of sinograms is the files acquired. However, this kind of files needs reconstruction in order to get an image with diagnostic value. The most known reconstruction methods are the Filtered Back-Projection (FBP) and the Iterative methods. Attenuation correction is resolved by using the constant linear attenuation coefficient $(\mu)$ method or using the transmission source method. In the first one, the distance that each photon has travelled is calculated based on the patient geometry and the exponential reduction of their intensity. Then, considering the human body as a uniform object, an attenuation map is implemented in the reconstructed image. The latter method utilises a transmission source which scans the patient. This depicts each pixel or voxel of the patient with a specific $\mu$ producing an attenuation coefficient map. Finally, the attenuation map isimplemented on the image resulting in a more accurate diagnosis. The second issue of scatter correction can be resolved by the electronics of the gamma camera and the filtering process during reconstruction. When a photon undergoes scattering, its energy reduces. So, a well defined function can accept for imaging photons with energy at a certain narrow energy window around the central photopeak of the $\gamma$ - emission. A hybrid SPECT/CT scanner is capable of implementing both a CT scan and a SPECT scan or it can be used for each of these scans separately.

Using the CT scan, the anatomy of a specific patient area can be imaged while the SPECT scan can depict the physiology of this area. Then, the registration of the two images drives at an image of advanced diagnostic value. Moreover, the CT data is used for the implementation of attenuation correction. (Delbeke et al., 2006) The range of nuclear medicine examinations is fairly wide. It includes, among others, patients' 
studies, as myocardium perfusion by $99 \mathrm{mTc}-$ Tetrofosmin or $99 \mathrm{mTc}$-Sestamibi, striatum imaging in brain by 123I-Ioflupane (DaTSCAN), renal parenchyma imaging by 99mTc-De-Methylo-Sulfo-Acid (DMSA) and 99mTc-Methylo-Di-Phosphonate (MDP) for bone scintigraphy. Fundamental image analysis methods of myocardium, brain, kidneys, thyroid, lungs and oncological (e.g. neuroblastoma) nuclear medicine studies include regions' properties, boundary analysis, curvature analysis or line and circle detection. Image processing serves in reconstruction of images acquired using SPECT techniques, in improvement of the quality of images for viewing and in preparation of images for quantitative results. Data of the mentioned examinations are used in the following applications of MatLab algorithms to make the image processing and analysis in nuclear medicine clear and show the MatLab utility for these studies.

\section{Image ANalysis ANd Processing In NuClear Medicine}

In the last several decades, medical imaging systems have advanced in a dynamic progress. There have been substantial improvements in characteristics such as sensitivity, resolution, and acquisition speed. New techniques have been introduced and, more specifically, analogue images have been substituted by digital ones.

As a result, issues related to the digital images' quality have emerged. The quality of acquired images is degraded by both physical factors, such as Compton scattering and photon attenuation, and system parameters, such as intrinsic and extrinsic spatial resolution of the gamma camera system. These factors result in blurred and noisy images. Most times, the blurred images present artefacts that may lead to a fault diagnosis.

In order the images to gain a diagnostic value for the physician, it is compulsory to follow a specific series of processing. Image processing is a set of techniques in which the data from an image are analysed and processed using algorithms and tools to enhance certain image information that is more useful to human interpretation (Nailon, 2010). The processing of an image permits the extraction of useful parameters and increases the possibility of detection of small lesions more accurately. Image processing in nuclear medicine serves three major purposes: a) the reconstruction of the images acquired with tomographic (SPECT) techniques.

b) the quality improvement of the image for viewing in terms of contrast, uniformity and spatial resolution and, c) the preparation of the image in order to extract useful diagnostic qualitative and quantitative information.

\section{* Digital images}

In all modern nuclear medicine imaging systems, the images are displayed as an array of discrete picture elements (pixels) in two dimensions (2D) and are referred as digital images. Each pixel in a digital image has an intensity value and a location address (Fig. 1). In a nuclear medicine image the pixel value shows the number of counts recorded in it. The benefit of a digital image compared to the analogue one is that data from a digital image are available for further computer processing.

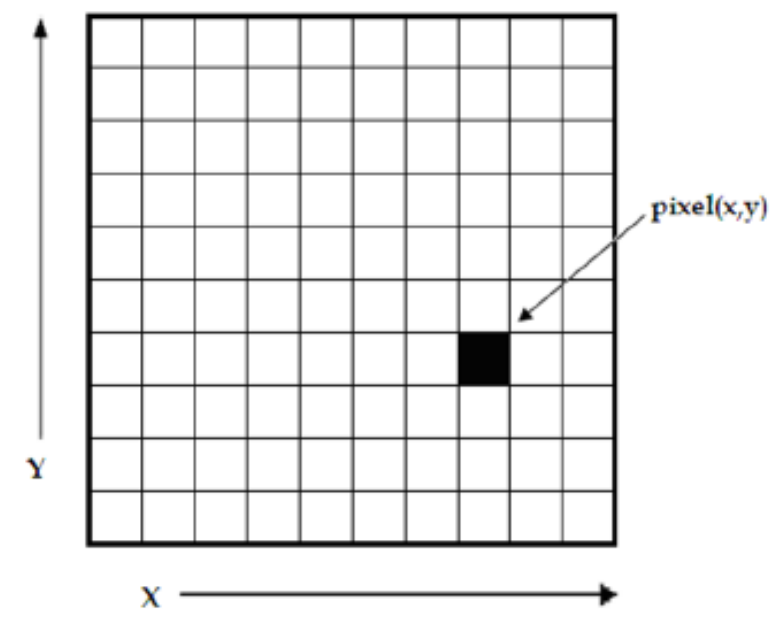

Fig. 1. A digital image is a 2D array of pixels. Each pixel is characterised by its (x,y) coordinates and its value.

Digital images are characterised by matrix size, pixel depth and resolution. The matrix size is determined from the number of the columns $(m)$ and the number of rows $(n)$ of the image matrix $(m \times n)$. The size of a matrix is selected by the operator. Generally, as the matrix dimension increases the resolution is getting better (Gonzalez et al., 2009). Nuclear medicine images matrices are, nowadays, ranged from $64 \times 64$ to $1024 \times 1024$ pixels. Pixel or bit depth refers to the number of bits per pixel that represent the colour levels of each pixel in an image. Each pixel can take $2 \mathrm{k}$ different values, where $\mathrm{k}$ is the bit depth of the image. This 
means that for an 8-bit image, each pixel can have from 1 to 28 (=256) different colour levels (grey-scale levels). Nuclear medicine images are frequently represented as 8- or 16- bit images. The term resolution of the image refers to the number of pixels per unit length of the image.

In digital images the spatial resolution depends on pixel size. The pixel size is calculated by the Field of View (FoV) divided by the number of pixels across the matrix. For a standard FoV, an increase of the matrix size decreases the pixel size and the ability to see details is improved.

\section{* Types of digital images - MatLab}

MatLab offers simple functions that can read images of many file formats and supports a number of colour maps. Depending on file type and colour space, the returned matrix is either a $2 \mathrm{D}$ matrix of intensity values (greyscale images) or a 3D matrix of RGB values. Nuclear medicine images are grey scale or true colour images (RGB that is Red, Green and Blue).

The image types supported from the Image Processing Toolbox are listed below:

- Binary Images. In these, pixels can only take 0 or 1 value, black or white.

- Greyscale or intensity images. The image data in a greyscale image represent intensity or brightness. The integers' value is within the range of $[0 \ldots 2 \mathrm{k}-1]$, where $\mathrm{k}$ is the bit depth of the image. For a typical greyscale image each pixel can represented by 8 bits and intensity values are in the range of [0..255], where 0 corresponds to black and 255

to white.

- True color or RGB. In these, an image can be displayed using three matrices, each one corresponding to each of red-green-blue colour. If in an RGB image each component uses 8 bits, then the total number of bits required for each pixel is $3 \times 8=24$ and the range of each individual colour component is [0...255].

- Indexed images. Indexed images consist of a $2 \mathrm{D}$ matrix together with an $\mathrm{m} \times 3$ colour map $(\mathrm{m}=$ the number of the columns in image matrix). Each row of map specifies the red, green, and blue components of a single colour.

An indexed image uses direct mapping of pixel values to colour map values. The colour of each image pixel is determined by using the corresponding value of matrix as an index into map.The greyscale image is the most convenient and preferable type utilised in nuclear medicine image processing. When colouring depiction is needed, the RGB one should be used and processed. The indexed type images should be converted to any of the two other types in order to be processed. The functions used for image type conversion are: rgb2gray, ind2rgb, ind2gray and reversely. Any image can be also transformed to binary one using the command: im2bw.

Moreover, in any image, the function impixelinfo can be used in order to detect any pixel value. The user can move the mouse cursor inside the image and the down left corner appears the pixel identity (x, y) as well as the (RGB) values. The pixel range of the image can be displayed by the command imdisplayrange.

\section{* Image processing techniques - MatLab}

Image processing techniques include all the possible tools used to change or analyse an image according to individuals' needs. This subchapter presents the most widely performed image processing techniques that are applicable to nuclear medicine images. The examples used are mostly come from nuclear medicine renal studies, as kidneys' planar images and SPECT slices are simple objects to show the application of image processing MatLab tools.

\section{* Contrast enhancement}

One of the very first image processing issues is the contrast enhancement. The acquired image does not usually present the desired object contrast. The improvement of contrast is absolutely needed as the organ shape, boundaries and internal functionality can be better depicted. In addition, organ delineation can be achieved in many cases without removing the background activity. The command that implements contrast processing is the imadjust. Using this, the contrast in an image can be enhanced or degraded if needed. Moreover, a very useful result can be the inversion of colours, especially in greyscale images, where an object of interest can be efficiently outlined. The general function that implements contrast enhancement is the following:

$\mathrm{J}=$ imadjust(I,[low_in high_in],[low_out high_out],gamma);

while the function for colour inversion is the following:

$\mathrm{J}=$ imadjust(I,[0 1],[1 0],gamma); or $\mathrm{J}=$ imcomplement(I);

suppose that $\mathrm{J}$, is the new image, $\mathrm{I}$, is the initial image and gamma factor depicts the shape of the curve that describes the relationship between the values of I and J. If the gamma factor is omitted, it is considered to be 1 .

\section{* Organ contour}

In many nuclear medicine images, the organs' boundaries are presented unclear due to low resolution or presence of high percentage of noise. In order to draw the contour of an organ in a nuclear medicine image, 
the command imcontour is used. In addition, a variable $\mathrm{n}$ defines the number of equally spaced contoursrequired. This variable is strongly related with the intensity of counts. For higher $n$ values, the lines are drawn with smaller spaces in between and depict different streaks of intensity. The type of line contouring can be specified as well. For example, when a contour of 5 level contours, drawn with solid line, is the desirable outcome, the whole function is:

Example 1I = imread('kindeys.jpg');

figure, imshow(I)

$\mathrm{J}=$ imcontour(I,5,'-`);

Figure, imshow $(\mathrm{J})$

Where $J$ and I stands for the final and the initial image respectively and the symbol ('-') stands for the solid line drawing. An example of the initial image, the contour with $n=15$ and $n=5$ respectively, follows.
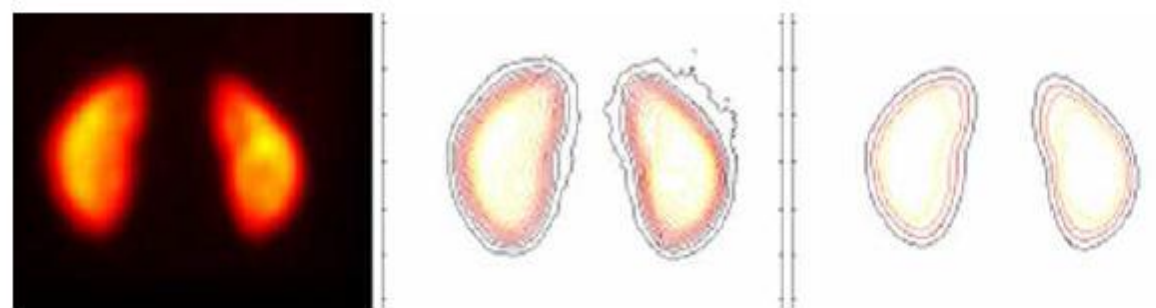

Fig. 2. (a) Original image depicting kidneys, (b) organs contoured with $n=15$, (c) organs contoured with $n=5$.

IV.

IMAGE INTERPOLATION

Interpolation is a topic that has been widely used in image processing. It constitutes of the most common procedure in order to resample an image, to generate a new image based on the pattern of an existing one. Moreover, re-sampling is usually required in medical image processing in order to enhance the image quality or to retrieve lost information after compression of an image (Lehmann et al., 1999). Interpreting the interpolation process, the user is provided with several options. These options include the resizing of an image according to a defined scaling factor, the choice of the interpolation type and the choice of low-pass filter. The general command that performs image resizing is imresize. However, the way that the whole function has to be written depends heavily on the characteristics of the new image. The size of the image can be defined as a scaling factor of the existing image or by exact number of pixels in rows and columns. Concerning the interpolation types usually used in nuclear medicine, these are the following: a) nearest-neighbour interpolation ('nearest'), where the output pixel obtains the value of the pixel that the point falls within, without considering other pixels, b) bilinear interpolation ('bilinear'), where the output pixel obtains a weighted average value of the nearest $2 \times 2$ pixels, c) cubic interpolation ('bicubic'), where the output pixel obtains a weighted average value of the nearest $4 \times 4$ pixels (Lehmann et al., 1999). When an image has to resize in a new one, with specified scaling factor and method, then the function Implementing that, is the following:

NewImage $=$ imresize(Image, scale, method);

For example, for a given image I, the new image $\mathrm{J}$ shrunk twice of the initial one, using the bilinear interpolation method, the function will be: $\mathrm{J}=$ imresize(I, 0.5 , 'bilinear');

This way of image resizing contributes to the conversion of image information during any such process, a fact that is valuable in the precision of a measurement. Bilinear interpolation is often used to zoom into a $2 \mathrm{D}$ image or for rendering, for display purposes. Apart from the previous methods, the cubic convolution method can be applied to 3D images.

\section{IMAGE FILTERING}

The factors that degrade the quality of nuclear medicine images result in blurred and noisy images with poor resolution. One of the most important factors that greatly affect the quality of clinical nuclear medicine images is image filtering. Image filtering is a mathematical processing for noise removal and resolution recovery. The goal of the filtering is to compensate for loss of detail in an image while reducing noise. Filters suppressed noise

as well as deblurred and sharpened the image. In this way, filters can greatly improve the image resolution and limit the degradation of the image. An image can be filtered either in the frequency or in the spatial domain. In the first case the initial data is Fourier transformed, multiplied with the appropriate filter and then taking the inverse Fourier transform, re-transformed into the spatial domain. The basics steps of filtering in the frequency domain are illustrated in Fig. 3. 


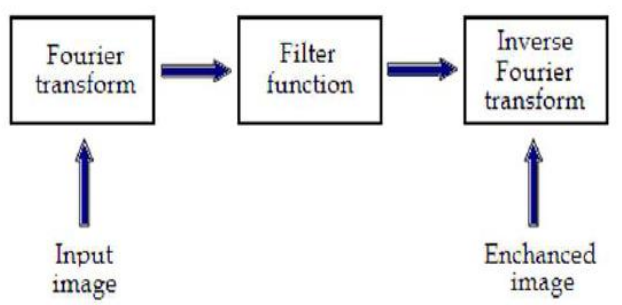

Fig. 3. Basics steps of frequency domain filtering.

The filtering in the spatial domain demands a filter mask (it is also referred as kernel or convolution filter). The filter mask is a matrix of odd usually size which is applied directly on the original data of the image.

The mask is centred on each pixel of the initial image. For each position of the mask the pixel values of the image is multiplied by the corresponding values of the mask. The products of these multiplications are then added and the value of the central pixel of the original image is replaced by the sum. This must be repeated for every pixel in the image. The procedure is described schematically in Fig. 3. If the filter, by which the new pixel value was calculated, is a linear function of the entire pixel values in the filter mask (e.g. the sum of products), then the filter is called linear. If the output pixel is not a linear weighted combination of the input pixel of the image then the filtered is called non-linear. According to the range of frequencies they allow to pass through filters can be classified as low pass or high pass. Low pass filters allow the low frequencies to be retained unaltered and block the high frequencies. Low pass filtering removes noise and smooth the image but at the same time blur the image as it does not preserve the edges. High pass filters sharpness the edges of the image (areas in an image where the signal changes rapidly) and enhance object edge information. A severe disadvantage of high pass filtering is the amplification of statistical noise present in the measured counts. The next section is referred to three of the most common filters used by MatLab: the mean, median and Gaussian filter.

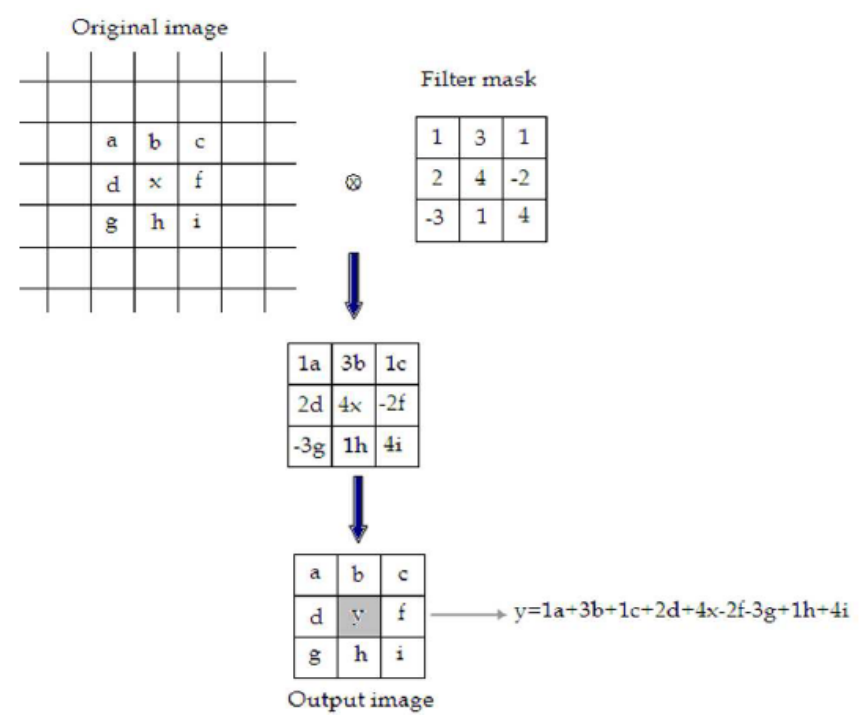

Fig. 4. Illustration of filtering process in spatial domain.

\section{* Mean filter}

Mean filter is the simplest low pass linear filter. It is implemented by replacing each pixel value with the average value of its neighbourhood. Mean filter can be considered as a convolution filter. The smoothing effect depends on the kernel size. As the kernel size increases, the smoothing effect increases too. Usually a $3 \times 3$ (or larger) kernel filter is used. An example of a single $3 \times 3$ kernel is shown in the Fig. 5 .

\begin{tabular}{|l|l|l|}
\hline$a$ & $b$ & $c$ \\
\hline$d$ & $e$ & $f$ \\
\hline$g$ & $h$ & $i$ \\
\hline
\end{tabular}

$$
\longrightarrow \frac{1}{9}(a+b+c+d+e+f+g+h+i)
$$

Fig. 5. Filtering approach of mean filter.

The Fig. 5 depicts that by using the mean filter, the central pixel value would be changed from "e" to "' $(\mathrm{a}+\mathrm{b}+\mathrm{c}+\mathrm{d}+\mathrm{e}+\mathrm{f}+\mathrm{g}+\mathrm{h}+\mathrm{i}) 1 / 9$ ". 


\section{* Median filter}

Median filter is a non linear filter. Median filtering is done by replacing the central pixel with the median of all the pixels value in the current neighbourhood. A median filter is a useful tool for impulse noise reduction (Toprak \& Göller, 2006). The impulse noise (it is also known as salt and paper noise) appears as black or (/and) white pixels randomly distributed all over the image. In other words, impulse noise corresponds to pixels with extremely high or low values. Median filters have the advantage to preserve edges without blurring the image in contrast to smoothing filters.

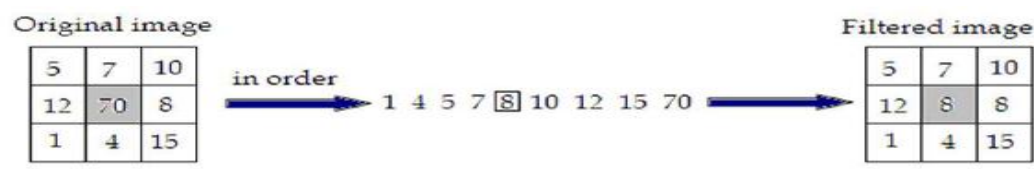

Fig. 6. Filtering approach of Median Filter.

\section{* Gaussian filter}

Gaussian filter is a linear low pass filter. A Gaussian filter mask has the form of a bellshaped curve with a high point in the centre and symmetrically tapering sections to either side (Fig.6). Application of the Gaussian filter produces, for each pixel in the image, a weighted average such that central pixel contributes more significantly to the result than function (Eq.1):

pixels at the mask edges (O'Gorman et al., 2008). The weights are computed according to the Gaussian

$$
f(x)=\frac{1}{\sigma \sqrt{2 \pi}} e^{-(x-\mu)^{2} /\left(2 \sigma^{2}\right)}
$$

where $\mu$, is the mean and $\sigma$, the standard deviation.

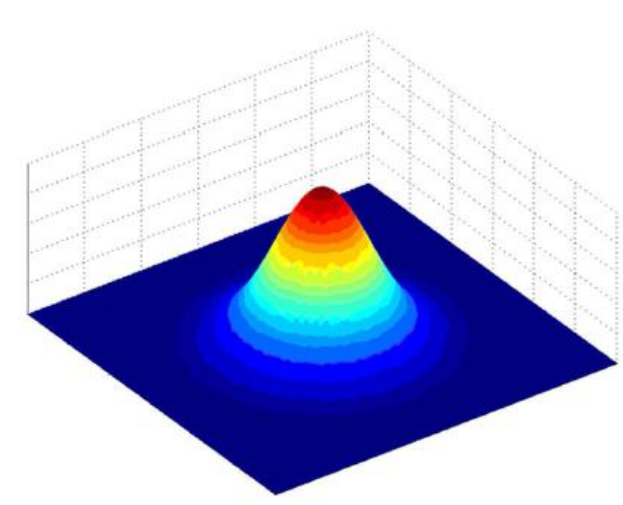

Fig. 7. A 2D Gaussian function.

The degree of smoothing depends on the standard deviation. The larger the standard deviation, the smoother the image is depicted. The Gaussian filter is very effective in the reduction of impulse and Gaussian noise. Gaussian noise is caused by random variations in the intensity and has a distribution that follows the Gaussian curve.

\section{IMAGE SEGMENTATION}

The image segmentation describes the process through which an image is divided into constituent parts, regions or objects in order to isolate and study separately areas of special interest. This process assists in detecting critical parts of a nuclear medicine image that are not easily displayed in the original image. The process of segmentation has been developed based on lots of intentions such as delineating an object in a gradient image, defining the region of interest or separating convex components in distance-transformed images.

Attention should be spent in order to avoid 'over-segmentation' or 'under-segmentation'. In nuclear medicine, segmentation techniques are used to detect the extent of a tissue, an organ, a tumour inside an image, the boundaries of structures in cases that these are ambiguous and the areas that radiopharmaceutical concentrate in a greater extent. Thus, the segmentation process serves in assisting the implementation of other procedures; in other words, it constitutes the fundamental step of some basic medical image processing (Behnaz et al., 2010). There are two ways of image segmentation:

a) based on the discontinuities and, 
b) based on the similarities of structures inside an image. In nuclear medicine images, the discontinuity segmentation type finds more applications. This type depends on the detection of discontinuities or else, edges, inside the image using a threshold.

The implementation of threshold helps in two main issues:

i) the removal of unnecessary information from the image (background activity) and,

ii) the appearance of details not easily detected. The edge detection uses the command edge.

In addition, a threshold is applied in order to detect edges above defined grey-scale intensity. Also, different methods of edge detection can be applied according to the filter each of them utilises. The most useful methods in nuclear medicine are the 'Sobel', 'Prewitt', 'Roberts', 'Canny' as well as 'Laplacian of Gaussian'. It is noted that the image is immediately transformed into a binary image and edges are detected. The general function used for the edge detection is the following:

$[\mathrm{BW}]=$ edge (image, 'method', threshold) Where $[\mathrm{BW}]$ is the new binary image produced, image is the initial one; 'method' refers to the method of edge detection and 'threshold' to the threshold applied. In nuclear medicine, the methods that find wide application are the sorbel, prewitt and canny. In the following example, the canny method is applied in order to detect edges in an image.

Example 4

I = imread('kidneys.jpg');

figure, imshow(I)

$\mathrm{J}=$ edge(I,'canny', 0.048);

figure, imshow $(\mathbf{J})$

Another application of segmentation in nuclear medicine is the use of gradient magnitude. The original image is loaded. Then, the edge detection method of sobel is applied in accordance with a gradient magnitude which gives higher regions with higher grey-scale intensity. Finally, the foreground details are highlighted and segmented image of the kidneys is produced. The whole code for that procedure is described below.

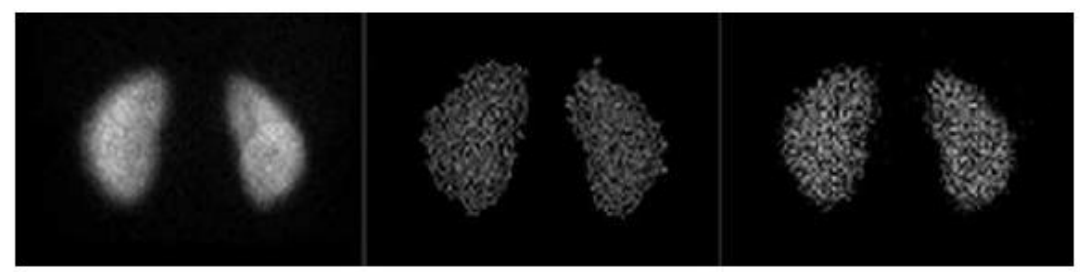

Fig. 8. Edge detection (a) Original kidneys image, (b) edge detection with canny method and threshold 0.2667, (c) edge detection with prewitt method and threshold 0.038. [(a) to (c) from left to right]

Example

I = imread('kidneys.jpg');

Figure, imshow(I)

hy = fspecial('sobel');

hx $=$ hy';

Iy = imfilter(double(I), hy, 'replicate');

Ix = imfilter(double(I), hx, 'replicate');

gradmag $=\operatorname{sqrt}\left(\operatorname{Ix} .{ }^{\wedge} 2+\operatorname{Iy} . \wedge 2\right)$;

figure, imshow(gradmag,[])

se $=$ strel('disk', 20);

$\mathrm{K}=$ imopen(I, se);

figure, imshow(K).
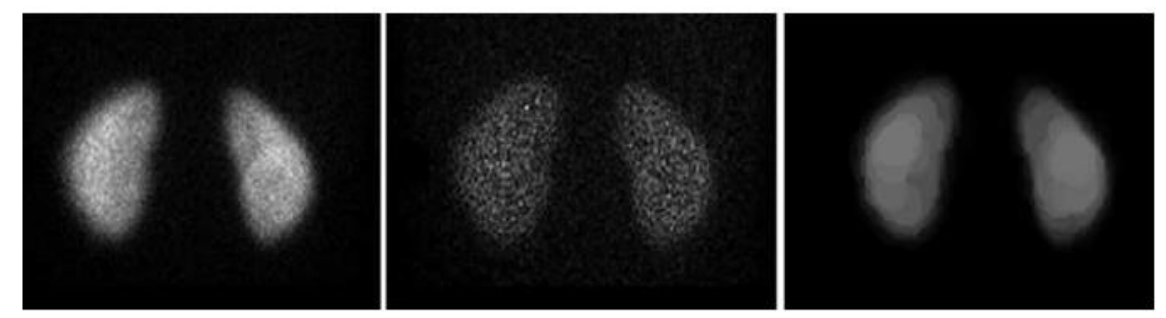

Fig. 9. Gradient Magnitude process: (a) Original image, (b) image after implementation of filter and gradient magnitude, (c) image after masking of foreground objects [(a) to (c) from left to right]

In the final image, the outline of the organs is depicted. The area inside the kidney has been separated into larger parts with grey-scale intensity weighted and decided from the closest 20 pixels in a circular region. In 
the areas of kidney that have higher activity concentrated, more than one layer of circular regions have been added presenting a final lighter region.

\section{BACKGROUND ACTIVITY REMOVAL}

One of the first steps to be completed in the medical image processing is removing the background activity. This procedure is based on image segmentation as in order to achieve the background activity removal, the organs' boundaries are first defined. The steps in this procedure are the following:

i) the image is read,

ii) the image is appeared,

iii) a grey level threshold is decided by MatLab,

iv) the image is transformed into binary image in order to isolate the two kidneys,

v) the binary image is multiplied by the initial one,

vi) the final image is appeared,

vii) the colour can change (or not) according to individuals' needs. The following example of kidneys image describes the process.

Example

I = imread('kidneys.jpg');

figure, imshow(I) (fig.10a)

graythresh(I) and the value of the threshold is calculated: ans $=0.2667$

$\mathrm{I} 2=\mathrm{im} 2 \mathrm{bw}(\mathrm{I}, 0.2667)($ fig. $10 \mathrm{~b})$

$\mathrm{I} 3$ = immultiply $(\mathrm{I} 2, \mathrm{I})$

imshow(I3) (fig.10c)

colormap(hot) (fig.10d)
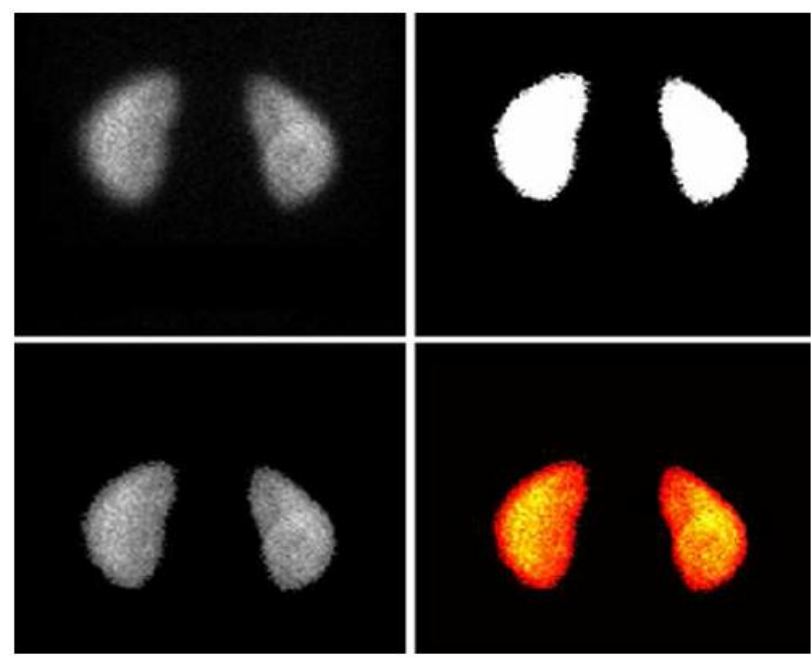

Fig. 10. Background subtraction: (a) Original image, (b) segmented binary image after thresholding depicting only sharp organ boundaries, (c) image after background removal,(d) change of colour to nuclear medicine pattern. [(a) to $(d)$ from left to right]

\section{VIII.}

\section{IMAGE REGISTRATION}

Image registration is used for aligning two images of the same object into a common coordinate system presenting the fused image. The one image is usually referred as reference and the other as sensed (or referred).

Image registration is a spatial transform. The images can be acquired from different angles, at different times, by different or same modalities. A typical example of the use of image registration from different modalities in nuclear medicine is the combination of SPECT and CT images (SPECT/CT) or PET and CT (PET/CT). Image registration is used mainly for two reasons: i) to obtain enhanced information and details from the image for more accurate diagnosis or therapy (Li \& Miller, 2010) and, ii) to compare patient's data (Zitova $\&$ Flusser, 2003). MatLab can be used in order to perform such a process. The whole procedure shall follow a specific order.

The first step of the procedure includes the image acquisition. After that, each image is reconstructed separately. Any filters needed are applied as well as enhancements in brightness and contrast. The process of filter application has been described in a previous section. The next step includes the foundation of a spatial transformation between the two images, the one of SPECT and the other of CT. The key figure in this step concerns about the alignment of the two images. A spatial transformation modifies the spatial relationship between the pixels of an image relocating them to new positions in a new image. There are several types of 
spatial transformation including the affine, the projective the box and the composite (Delbeke et al., 2006). The final step in image registration is the overlapping of the two images allowing a suitable level of transparency. A new image is created containing information from both pictures from which, the first has been produced. The whole procedure can be described with a set of commands which is user customised as different registration function packages can be constructed for different uses.

\section{ARthroplasty - General Presentation}

Arthroplasty [10] represents a surgical procedure in which the arthritic or dysfunctional joint surface is replaced with prosthesis or by remodeling or realigning the joint. The important joint for this article is the one located at hip area. This is the reason why the article details the parameters that belong to the thigh-bone and the pelvis. Fig. 11 presents the most important parameters in Hip Arthroplasty, extracted from an anterior-posterior radiography. Fig. 12 presents the parameters extracted from an anterior-lateral radiography representing the hip after the insertion of the prosthesis.For the scope of this article, three areas of the thighbone are analyzed: the femoral head (the nearest part to the pelvis), the femoral neck, and the femoral shaft or body (the longest part of the thigh-bone).

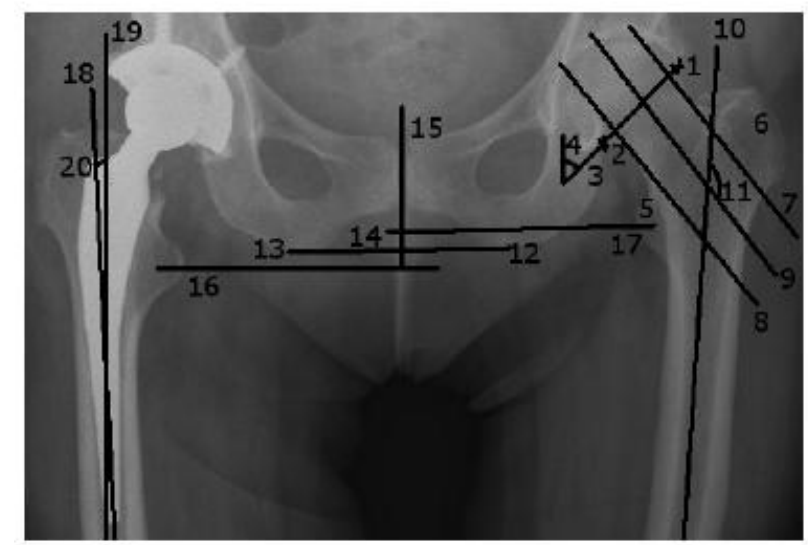

Fig.11. Parameters important in Hip Replacement, extracted from an anterior-posterior radiography

The parameters of interest for the Hip Replacement [7] are listed below:

1) the superior margin of the acetabulum (the superior point in which the thigh-bone meets the pelvis).

2) the inferior margin of the acetabulum (the inferior point in which the thigh-bone (the femoral head) meets the pelvis).

3) the femoral head axis or the acetabulum axis (the line determined by the two points that represent the superior margin and the inferior margin of the acetabulum).

4) the angle created by the acetabulum axis with the vertical line. This angle has to be the same for both of the femoral bones.

5) the lesser trochanter (located in the upper left part of the femoral body).

6) the greater trochanter (located in the upper right part of the femoral body).

7) the tangent to the superior cortical of the femoral neck

8) the tangent to the inferior cortical of the femoral neck

9) the femoral neck axis (the axis of the cylinder determined by the tangents 7 and 8)

10) the femoral body axis or the diaphyseal axis (the axis of the cylinder that approximates the femoral body)

11) the most important parameter, extracted from the $x$-ray before the surgical intervention is represented by the cervicodiaphyseal angle (the angle determined by the neck axis and the diaphyseal axis).Depending on the value of this angle, it can be determined whether the patient needs or not a prosthesis. If the angle has values between 125 and 135 degrees, the thigh-bone is considered to be in normal ranges.

12) the right ischiadic tuberosity (the lowest right part of the pelvic bone).

13) the left ischiadic tuberosity (the lowest left part of the pelvic bone).

14) the ischiadic line or the horizontal reference line (the line determined by the two ischiadic tuberosities).

15) the vertical reference line, that is perpendicular on the ischiadic line, in its middle.

16) the line starting from the center of the lesser left trochanter, parallel to the ischiadic line

17) the line starting from the center of the right lesser trochanter, parallel to the ischiadic line; the distance between lines 16 and 17 represents the vertical distance between the two thigh-bones. If this distance is greater 
than a chosen threshold, there is an indicationof a difference between the lengths of the two femoral bones that has to be resolved surgically (in most cases).

After inserting the prosthesis, some new parameters must be taken into consideration:

18) the diaphyseal axis of the femoral bone (the same as parameter 10).

19) the diaphyseal axis of the prosthesis or the axis of the prosthesis' body (the axis of the

cylinder that approximates the prosthesis' body).

20) The deviation of the prosthesis (the angle determined by lines 18 and 19).

This parameter will be computed in several radiographic images, following the evolution of the same patient. The important parameters extracted from an anteriorlateral radiography, after inserting the prosthesis, are shown in Fig.12:

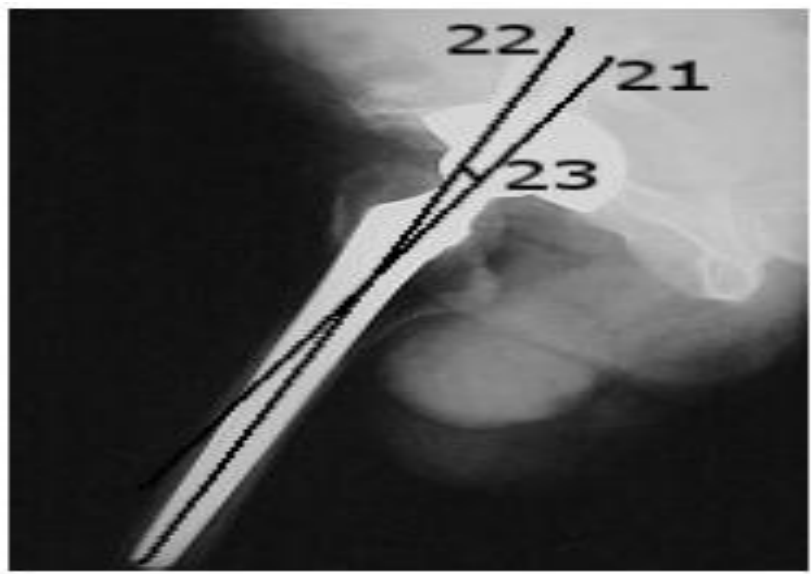

Fig.12. Parameters important in Hip Replacement,extracted from an anterior-lateral radiography, after the insertion of the prosthesis.

21) The axis of the prosthesis' neck

22) The axis of the prosthesis' body

23) The anteversion angle (the angle determined by the axes 21 and 22).

If this angle has its value situated between 5 and 10 degrees, it's considered to be in normal ranges.

\section{The Dicom Standard}

In order to manage and interpret the x-rays data in a simple and organized manner, a standard for the $\mathrm{x}$-ray files is needed. This is the reason why the most popular standard for medical images was chosen: DICOM [14]. The Digital Imaging and Communications in Medicine(DICOM) standard is a detailed specification of the coding and transfer of medical images and their associated information.

\section{* Representing Data In The DiCOM Format}

The clinical data are represented in a variety of formats:the distances are measured in millimeters, the time in seconds, etc. The PS 3.5 part of the standard, entitled. Data Structure and Their Encoding, defines 27 types of standard data, known as "value representations" (VR), that include all types of data that can appear in the medical domain. Any information encoded in a DICOM file has to belong to one of these predefined types.

Some of the most important standard data are: Person Name (PN), Date Time (DT), and Age String (AS).A DICOM file has the following structure:

- A preamble of 128 bytes

- A prefix (4 bytes) for retaining the letters ' $\mathrm{D}$ ', 'I',

'C', 'M' (the signature of a DICOM file)

- A data set that contains information like: patient's name, image type, image dimension, etc.

- Pixels that form the image (or the images) contained in the file.

\section{* Extracting Data From DiCOM Files}

Extracting data from a DICOM file can be made using the tags defined in the DICOM dictionary. Every tag is searched in the file, and, if found, is interpreted.

The steps in extracting the information are:

- Checking the existence of the characters ' $\mathrm{D}$ ', 'I',

'C', 'M'

- Determining of the VR type - Setting the order of the bytes (Big Endian or Little Endian)

- Searching for a tag in the DICOM file,corresponding to the order of bytes and the VR type 
- Extracting the values corresponding to that tag Some characteristics of the DICOM files, important when extracting data, are:

- the number of images contained in the DICOM file

- the number of bits per pixel: 8, 12, 16, 24

- the compression

- the photometric interpretation: shades of gray or color images In case of images without compression, the extraction of the images is made pixel by pixel, according to the

number of bits per pixel. For images with compression, a decompression step should be previously performed.

\section{THE STRUCTURE OF THE CR DICOM FILES}

Computer radiographic images (CR) stored in DICOM files are accompanied by general identification elements and some specific information. For example, the Patient module contains: the name of the patient, the patient's ID, the patient's date of birth,

etc. Another module, specific for CR, CR Series, contains information about the examined body part, the view position, etc. Our application extracts from every module the important elements for managing and interpreting the patient's data.

\section{* Radiographic Image Processing}

As in any image analysis application, the first step is a preprocessing step, needed to improve the image by noise removal, contrast improvement, edge enhancement and others [4]. In our application, this step is followed by a contour extraction step, which helps in the arthroplasty parameters' extraction.

\section{* Image Enhancement}

One reason why the automatic interpretation of radiographic images doesn't give accurate results is the fact that the radiographic images are blurred. This is why enhancing images before applying contour detection algorithms is a step that should not be omitted.In the case of our application, the radiographic images are enhanced by noise removal, edge enhancement and

contrast improvement. We will detail each method in the following subsections.

\section{* Contour Extraction}

Most of the contour extraction algorithms which are based on edge detection follow these steps:

- detecting the edge pixels (pixels where the intensity changes abruptly)- eliminating the edge pixels which are not also contour pixels - connecting the contour pixels using local methods (based on the pixels' relations to their neighbor pixels) or global methods (based on global information, for example the shape of a bone, in a computer radiography).After trying a series of methods, the Canny algorithm [5] has been chosen in order to extract the contour lines, because this produced the best results. The Canny algorithm will be briefly described in the following lines. The Canny edge detection algorithm is a very well known algorithm and is considered by many the optimal edge detector. The algorithm is structured into 6 steps:

1) filter out any noise in the original image with the Gaussian filter.

2) apply the Sobel operator on the resulting image, estimating the gradient in the horizontal direction $(G x)$ and in the vertical direction $(G y)$. The magnitude, or the edge strength is approximated by the sum between $G x$ and $G y$ 3) find the edge direction, as the arctangent of $G y / G x$.

4) Once the direction is known, relate the edge direction to a discretized direction (all the angles between 67.5 and 112.5 will be considered to be of 90 degrees, all angles between 112.5 and 157.5 are set to 135 degrees, etc). Fig. 13 shows the possible discretized edge directions, previously determined in step 3 .

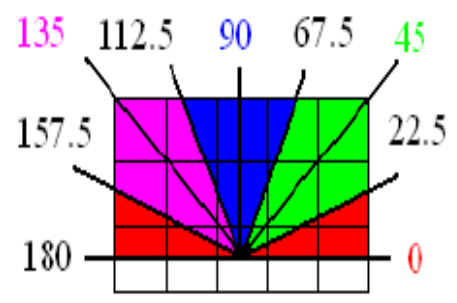

Fig. 13. Gradient direction

5) Non-maximum suppression (trace along the edge in the edge direction and suppress any pixel that is not considered to be an edge - that is not a local maximum)

6) Hysteresis for eliminating streaking, using two thresholds, $T 1$ (high) and $T 2$ (low). Any pixel with a value greater than $T l$ is considered to be an edge pixel. After applying the first threshold, any pixels connected 
to the edge pixels that have a value greater than $T 2$ will also be selected as edge pixels. Usually $T 1=2 * T 2$. The result of applying the Canny detector is a binary image (Fig. 14), where white pixels represent contour pixels. Having the contour lines of the bones, the next step in our application is the extraction of the important parameters in hip arthroplasty (automatic and semiautomatic extraction). We can observe in Fig. 14 that the contour is disconnected in some parts of the radiography, and that it cannot be reconstructed with accuracy. That is why we propose to search for some salient parameters (for example the lines representing the contour of the femoral body) that can be identified without the previous reconstruction of the entire pelvic and femoral contour. In the following section we will present the methods used after preprocessing the image (with noise removal, edge enhancement, contrast improvement and Canny edge detection), in order to extract the parameters important in arthroplasty.

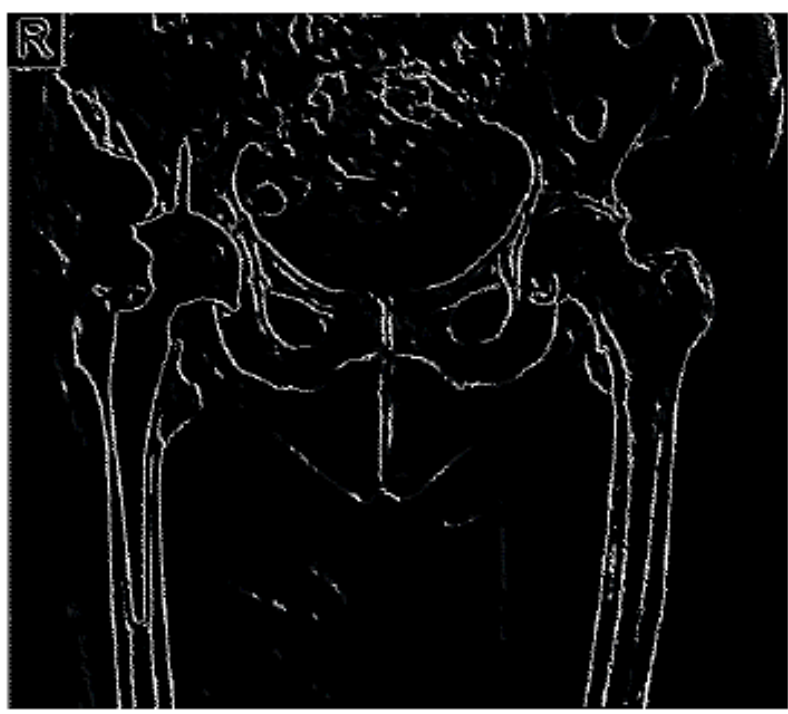

Fig.14. The image after applying the Canny Edge Detector

\section{CONCLUSION}

The research in the field of medical image analysis is a continuous challenge. The need to discover new image analysis algorithms and new automatic learning techniques that would help in computer assisted diagnosis is and will be a topic of interest for researchers.

The results of our research, presented in this paper, prove that solutions do exist. Although not all the arthroplasty parameters determined automatically were $100 \%$ accurate, the application proved to be very useful to doctors. The fact that the application allows patient's data saving management, during a long period of time after the hip replacement procedure is another plus. This application can be used for a single hospital, or for an entire national/international network of hospitals, integrating other applications of diagnosis or of assisting doctors in planning certain surgeries and following the patients' evolution after the surgeries. Image processing and analysis applied to nuclear medicine images for diagnosis, improve the acquired image qualitatively as well as offer quantitative information data useful in patient's therapy and care. Advanced techniques of image processing and analysis find widespread use in nuclear medicine. MatLab and Image Processing Toolbox enable both quantitative analysis and visualization of Nuclear Medicine images acquired as planar or angle projected images to reconstruct tomographic (SPECT, PET) slices and 3D volume surface rendering images.

\section{REFERENCES}

[1] Bankman I, Handbook of Medical Image Processing and Analysis, Academic Press, 2000.

[2] Feng D D, Biomedical Information Technology,Elsevier, 2008

[3] Chen Y, Ee X, Leow K W, Howe T S, Automatic Extraction of Femur Contours from Hip X-ray images, 2000.

[4] Gonzales R C, Woods R E, Digital Image Processing, Prentice-Hall, 2002

[5] Canny J F, A Computational Approach to Edge Detection, IEEE Trans. Pattern Analysis and Machine Intelligence, 1986/

[6] Campilho A, Kamel M, Image Analysis and Recognition Springer.

[7] Raj K Sinha, Hip Replacement.

[8] Kennon R, Hip and Knee Surgery: A Patient's Guide to Hip Replacement, Hip Resurfacing, Knee Replacement, and Knee Arthroscopy Book Description.

[9] Botez P, Ortopedie, Bit Publishing House (Iasi), 2001.

[10] Morrey B F, Joint Replacement Arthtoplasty,

[11] SCANIP: Available at http://www.simpleware.com/software/scanip/iptechd ata.php in 10.10.2009 
[12] 3D-DOCTOR: Available at http://www.ablesw.com/3d-doctor/index.html in 10.10.2009

[13] Hip-OpCT: Available at http://www.hipop.it/hipopct.html in 20.10.2009

[14] The official page of the DICOM standard. Available at http://dicom.nema.org in 20.05.2009

[15] Mohapatra S, Kumar Sa P, Majhi B, Impulsive Noise Removal Image Enhancement Technique, $6^{\text {th }}$ WSEAS International Conference on CIRCUITS, SYSTEMS, ELECTRONICS, CONTROL \& SIGNAL PROCESSING, Cairo, Egypt, 2007.

[16] Chen T, Wu HR, Adaptive impulse detection using center weighted median filters, IEEE Signal Process Lett, 2001

[17] Georgakopoulos S, Andreadis A, Image Noise Removal Using Graph Theory Concepts, WSEAS Multiconference: Signal, Speech and Image Processing, Rethymno, Greece, 2003

[18] Mohammad F, Al-Otum H M, Oraiqat M T, A Comparison of Image Enhancement using Curvelet Transform with Multiscale Gradient and Retinex Operators, WSEAS Multiconference: Signal, Speech and Image Processing, Izmir, Turkey, 2004

[19] Djekoune O, Achour K, Halimi M, Kahlouche S, Incremental Hough Transform: An Improvement Algorithm for Digital Devices Implementation, WSEAS Int. Conference on Electronics, Control \& Signal Processing and E-Activities, Singapore, 200

[20] Bidgood, D. \& Horii, S. (1992). Introduction to the ACR-NEMA DICOM standard. RadioGraphics, Vol. 12, (May 1992), pp. (345$355)$

[21] Delbeke, D.; Coleman, R.E.; Guiberteau M.J.; Brown, M.L.; Royal, H.D.; Siegel, B.A.; Townsend, D.W.; Berland, L.L.; Parker, J.A.; Zubal, G. \& Cronin, V. (2006). Procedure Guideline for SPECT/CT Imaging 1.0. The Journal of Nuclear Medicine, Vol. 47, No. 7, (July 2006), pp. (1227-1234). Gonzalez, R.; Woods, R., \& Eddins, S. (2009) Digital Image Processing using MATLAB, (second edition), Gatesmark Publishing, ISBN 9780982085400, United States of America

[22] Lehmann, T.M.; Gönner, C. \& Spitzer, K. (1999). Survey: Interpolation Methods in Medical Image Processing. IEEE Transactions on Medical Imaging, Vol.18, No.11, (November 1999), pp. (1049-1075), ISSN S0278-0062(99)10280-5

[23] Lyra, M.; Sotiropoulos, M.; Lagopati, N. \& Gavrilleli, M. (2010a). Quantification of Myocardial Perfusion in 3D SPECT images Stress/Rest volume differences, Imaging Systems and Techniques (IST), 2010 IEEE International Conference on 1 -2 July 2010, pp 31 - 35, Thessaloniki, DOI: 10.1109/IST.2010.5548486

[24] Lyra, M.; Striligas, J.; Gavrilleli, M. \& Lagopati, N. (2010b). Volume Quantification of I-123 DaTSCAN Imaging by MatLab for the Differentiation and Grading of Parkinsonism and Essential Tremor, International Conference on Science and Social Research, Kuala

[25] Lumpur, Malaysia, December 5-7, 2010. http://edas.info/p8295 Li, G. \& Miller, R.W. (2010). Volumetric Image Registration of Multi-modality Images of CT,

[25] MRI and PET,Biomedical Imaging, Youxin Mao (Ed.), ISBN: 978-953-307-071-1, InTech,Available from:http://www.intechopen.com/articles/show/title/volumetric-image-registrationof- multi-modality-images-of-ct-mri-and-pet

[26] O' Gorman, L.; Sammon, M. \& Seul M. (2008). Practicals Algorithms for image analysis, (second edition), Cambridge University Press, 978-0-521-88411-2, United States of America

[27] Nailon, W.H. (2010). Texture Analysis Methods for Medical Image Characterisation, Biomedical Imaging, Youxin Mao (Ed.), ISBN: 978-953-307-071-1, InTech, Available from: http://www.intechopen.com/articles/show/title/texture-analysis-methodsfor-

[28] medical-image-characterisationMathWorks Inc. (2009) MATLAB User's Guide. The MathWorks Inc., United States ofAmerica.

[29] Perutka K. (2010). Tips and Tricks for Programming in Matlab, Matlab - Modelling, Programming and Simulations, Emilson Pereira Leite (Ed.), ISBN: 978-953-307-125-1, InTech, Available from: http://www.intechopen.com/articles/show/title/tipsandtricks-for-programming-in-matlab.

[30] Toprak, A. \& Guler, I. (2006). Suppression of Impulse Noise in Medical Images with the Use of Fuzzy Adaptive Median Filter. Journal of Medical Systems, Vol. 30, (November2006), pp. (465-471)

[31] Wernick, M. \& Aarsvold, J. (2004). Emission Tomography: The Fundamentals of PET and SPECT,Elsevier Academic Press, ISBN 0-12-744482-3, China Wilson, H.B.; Turcotte, L.H. \& Halpern, D. (2003). Advanced Mathematics and Mechanics Applications Using MATLAB (third edition), Chapman \& Hall/CRC, ISBN 1-58488-262-X, United States of America

[32] Zitova, B. \& Flusser J. (2003). Image Registration methods: a survey. Image and Vision Computing. Vol 21, (June 2003), pp. (9771000). 